\title{
Argon Ion and Neutral Metastable Levels Destruction in a Magnetically Enhanced Inductively Coupled Plasma Reactor
}

\author{
T. Bieber ${ }^{1,2}$, X. Glad ${ }^{*}, 1,2$ L. de Poucques ${ }^{1,2}$, R. Hugon ${ }^{1,2}$, J.-L. Vasseur ${ }^{1,2}$ and J. Bougdira ${ }^{1,2}$ \\ ${ }^{1}$ Université de Lorraine, Institut Jean Lamour, Vandoeuvre-lès-Nancy, F-54506, France \\ ${ }^{2}$ CNRS, Institut Jean Lamour, Vandoeuvre-lès-Nancy, F-54506, France
}

\begin{abstract}
This paper deals with pure argon plasma studied in a magnetically enhanced inductively coupled reactor. Laser induced fluorescence technique was performed with an optical parametric oscillator laser on the $3 d^{4} F_{7 / 2}$ and $3 d^{2} G_{9 / 2}$

$\mathrm{Ar}^{+}$metastable levels and on the $\left(2 P_{3 / 2}^{0}\right) 4 s^{2}$ neutral metastable state to determine their relative densities. Langmuir probe was used as a complementary diagnostic to measure the electron energy distribution functions. When increasing the confinement magnetic field, the metastable state density collapses for all cited levels, by contrast with $n_{e}$ which is continuously growing. Calculations were carried out for neutral argon to explain this behaviour. The results show that the metastable population is depleted by electron-impact excitation and ionization, these loss processes becoming dominant compared to the metastable state creation term.
\end{abstract}

Keywords: Argon metastable states, EEDF, ICP, Langmuir probe, LIF.

\section{INTRODUCTION}

Argon gas is commonly used in high-density plasma processing, whether in combination with molecular gases to increase plasma density and stabilize the discharge, or in sputtering processes. In consequence, it has been thoroughly studied in the past years both experimentally and numerically.

In argon plasmas, metastable levels of argon neutral and ion play a major role $[1,2]$, and one of the most fitted tool to study their behaviour is laser induced fluorescence (LIF). This technique was first used as a plasma diagnostic in the 1970s [3, 4], and has been widely employed to determine atom or molecule velocity distribution functions, densities and temperatures, with the main advantage of being spatially resolved. This technique was successfully performed to characterize ion or neutral species in inductively coupled plasmas (ICP) [5-7] helicon discharges [8, 9], electron cyclotron resonance (ECR) reactors $[10,11]$ and magnetron sputtering devices [12-14].

During the characterization in pure argon of a helicon configuration source designed to study chemical etching [15], tunable diode-laser induced fluorescence (TD-LIF) on the $3 d^{4} F_{7 / 2} \quad \mathrm{Ar}^{+}$metastable level showed a process of metastable depopulation when increasing confinement magnetic field in inductive mode. This phenomenon was assumed to be due to metastable state electron-impact excitation towards higher radiative levels.

\footnotetext{
*Address correspondence to this author at the Université de Lorraine, Institut Jean Lamour, Vandoeuvre-lès-Nancy, F-54506, France;

Tel: +33 (0)3.83.68.49.07; Fax: +33 (0)3 835843 47; E-mail: xavier.glad@univ-lorraine.fr
}

In the 1990s, several research teams published results on electron-impact excitation cross sections (Bretagne et al. [16], Vlcek [17], and were modified later by Bogaerts et al. [18] and Bultel et al. [19]). These databases were also complemented in the last decade [20-23]. The latter cross sections have enabled to deepen the knowledge of argon metastable kinetics in various conditions, such as in microwave discharges [24, 25], ICP [2], or helicon plasmas [26]. However, as far as we know, no numerical model explaining metastable quenching at fixed low pressure while varying confinement magnetic field could be found in the literature.

The present experimental study is focused on furthering the interpretation of this argon ion metastable state depopulation. We carried out LIF measurements with a tunable pulsed optical parametric oscillator (OPO) laser on the $3 d^{4} F_{7 / 2} \quad \mathrm{Ar}^{+}$metastable state to confirm the results of our previous work [15] where this depopulation phenomenon was first observed. LIF was also performed on the $3 d^{2} G_{9 / 2}$ $\mathrm{Ar}^{+}$and $\left(2 P_{3 / 2}^{0}\right) 4 s^{2}$ Ar metastable levels to determine if they also present a population depletion in similar conditions. Langmuir probe diagnostics measuring the electron energy distribution function (EEDF) complement these experiments. Finally, knowing that, unlike $\mathrm{Ar}^{+}$ metastable states, electron-impact cross sections are available for neutral argon levels, a calculation estimating neutral argon metastable density is presented. The latter enforces this assumption of a depopulation mainly by electron-impact excitation leading to a secondary ionization source from neutral metastable state. 


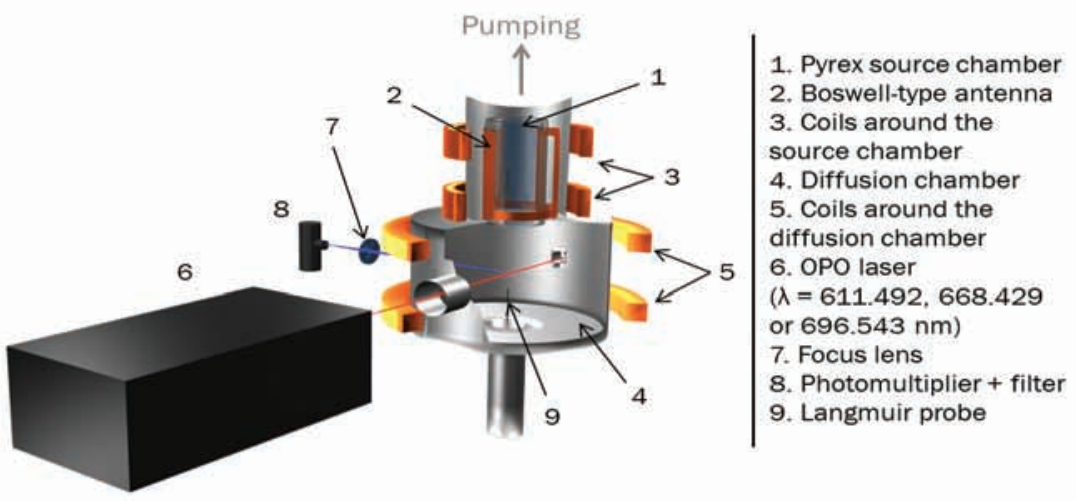

Fig. (1). Scheme of the helicon configuration reactor with the two diagnostics: LIF and Langmuir probe.

\section{MATERIALS AND METHODOLOGY}

\subsection{Plasma Source}

Experiments were performed in a helicon configuration reactor illustrated in Fig. (1) [27]. A $158-\mathrm{mm}$ diameter and $180-\mathrm{mm}$ high Boswell-type antenna [28] surrounds the Pyrex source chamber of an internal diameter of $132 \mathrm{~mm}$ and a height of $300 \mathrm{~mm}$. The former is connected to a $13.56-\mathrm{MHz}$ RF power supply via a $\Pi$ matching network. RF power was fixed at 500,700 and 900 W. A static magnetic field $B_{\text {source }}$ generated by copper coils can be applied in the source chamber up to $200 \mathrm{G}$.

The source chamber is connected to a $320-\mathrm{mm}$ diameter and $230-\mathrm{mm}$ high stainless steel diffusion chamber. Copper coils surrounding the latter can generate a confinement magnetic field $B_{\text {diff }}$ up to $100 \mathrm{G}$. Note that $B_{\text {source }}$ and $B_{\text {diff }}$ are oriented downwards, i.e. from source to diffusion chamber. Argon is injected in the diffusion chamber through a 50 -sccm mass flow controller and a solenoid valve, using a feedback loop from a Pfeiffer Vacuum CMR 264 capacitance gauge, keeps the working pressure constant.

Depending on experimental conditions, four different RF coupling modes are available in this plasma source [15]. At low RF power $\left(P_{R F}\right)$ and/or high pressure, capacitive mode is achieved. If $B_{\text {source }}$ is weak at low pressure and high RF power, inductive mode is obtained. In other cases, for higher values of $B_{\text {source }}$, the Trivelpiece-Gould (TG) mode occurs [29] due to the propagation of a TG wave along the Pyrex tube. Finally, for specific $P_{R F} / B_{\text {source }}$ values, helicon+TG mode is achieved [30]. The latter is characterized by the addition, with the TG mode, of a thin cylinder of luminous plasma following the diffusion chamber axis due to helicon wave propagation. One may note that since the power absorption occurs within the source chamber, the plasma creation volume, limited by the diameter of the tube $\left(\simeq 5000 \mathrm{~cm}^{3}\right)$, remains relatively constant with respect to the $\mathrm{RF}$ power and confinement magnetic field.

\subsection{Diagnostics}

Laser induced fluorescence was performed with a Continuum Sunlite pulsed optical parametric oscillator
(OPO) laser to obtain relative densities for several metastable states of argon ion and neutral [6, 31-33]. The use of such a solid laser enables to probe the three considered species in a row.

Fig. (1) shows the optical arrangement of such a diagnostic. The laser beam enters through the front window to excite the aimed species in the plasma. The OPO laser was tuned at 611.492 and $668.429 \mathrm{~nm}$ to probe the $3 d^{2} G_{9 / 2}$ and $3 d^{4} F_{7 / 2} \mathrm{Ar}^{+}$metastable levels (with lifetimes of 4.45 and $4.9 \mathrm{~s}$, respectively [34]). Subsequent fluorescence from the centre of the diffusion chamber is detected at right angle through a focal lens by a photomultiplier. The wavelength is selected with an interferential filter (1-nm width) centred at 460.9 or $442.6 \mathrm{~nm}$, respectively, as shown in Fig. (2). The obtained signal is then gate-integrated over the duration of the fluorescence signal, about $30 \mathrm{~ns}$, and averaged with a boxcar average-gated integrator from Stanford Research Systems.

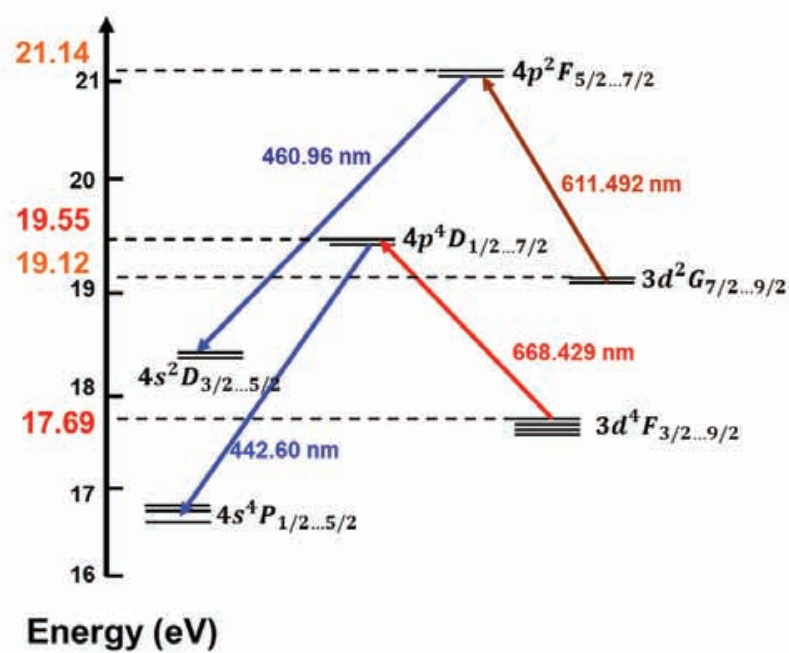

Fig. (2). Grotrian diagram of $\mathrm{Ar}^{+}$underlying the pumping and fluorescence transitions involved in LIF diagnostic.

The OPO laser was also tuned at $696.543 \mathrm{~nm}$ to probe the $\left(2 P_{3 / 2}^{0}\right) 4 s^{2}$ neutral metastable state (with a lifetime of 38 $\mathrm{s}$ [35]), as seen in Fig. (3), and the fluorescence was selected at $772.42 \mathrm{~nm}$ with a monochromator. 


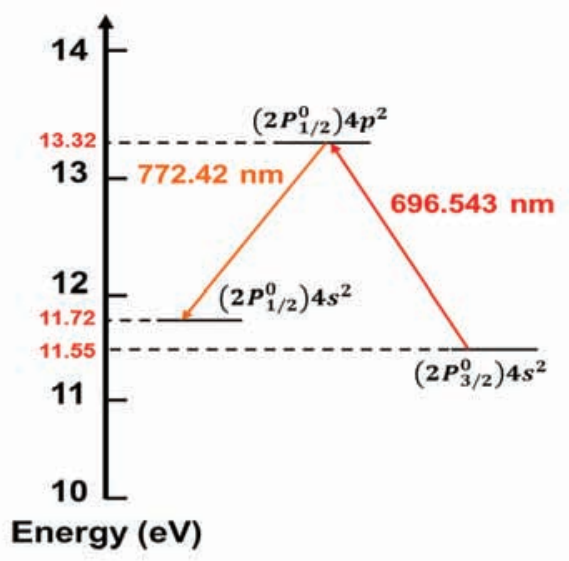

Fig. (3). Grotrian diagram of neutral Ar underlying the pumping and fluorescence transitions involved in LIF diagnostic.

Since the spectral resolution of the OPO laser is about 4 $\mathrm{GHz}$, which is of the same order of magnitude as the Doppler broadening for argon, Doppler profile cannot be obtained by OPO-LIF technique. It is possible by TD-LIF, as described in [15]. Thus, the OPO laser was tuned at the wavelength corresponding to the maximum of the detected LIF signal for each species. Fig. (4) shows a comparison between three types of LIF results for the same experiment. The first one presents the area under the line profile obtained by TD-LIF (1). This area is proportional to $\mathrm{Ar}^{+}$relative density, and is not affected by possible Zeeman line broadening, which increases with $B_{\text {diff }}$ up to $0.5 \mathrm{GHz}$, since the whole profile is integrated. The second type considers the maximum of the line intensity profile measured by TD-LIF (2). One can see that it presents the same behaviour as (1). This means that the line intensity is also proportional to $\mathrm{Ar}^{+}$ relative density and is only weakly affected by Zeeman effect. Consequently, the latter phenomenon cannot explain the significant decrease of the $\mathrm{Ar}^{+}$LIF signal by a factor of 10 (between 1.0 and around 0.1, as seen in Fig. 4).

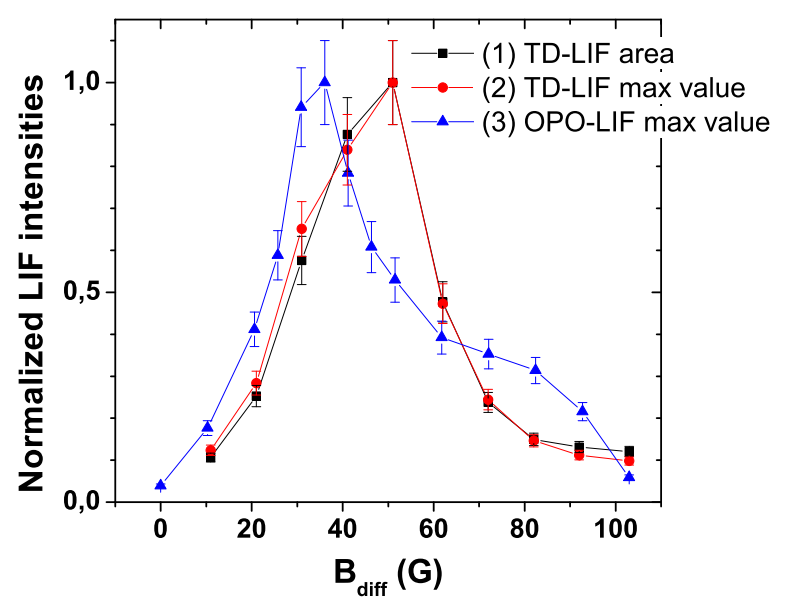

Fig. (4). Comparison between TD-LIF (area of the line profile (1) and maximum value of line intensity profile (2)) and OPO-LIF (maximum value of line intensity profile (3)) techniques. $P_{R F}=900$ $\mathrm{W}, B_{\text {source }}=0 \mathrm{G}$ and $\mathrm{P}=4 \mathrm{mTorr}$.
Finally, the line intensity maximum was measured by LIF with the OPO laser (3). Its behaviour is mostly similar to (1) and (2). The beam section is $\simeq 5 \mathrm{~mm}^{2}$ for TD-LIF and $\simeq 20 \mathrm{~mm}^{2}$ for OPO-LIF and we have estimated that the signal is integrated over a cylinder with a maximum length of $10 \mathrm{~mm}$. Therefore, these probed volumes are sufficiently small not to induce any discrepancy between the two techniques. The slight difference is probably due to the experimental conditions which cannot be exactly alike a few months later. In consequence, the maximum value of the OPO-LIF signal can be considered as representative of the probed metastable state behaviour $\left(\left[A r^{+, m}\right]_{r e l}^{668},\left[A r^{+, m}\right]_{r e l}^{611}\right.$ or $\left[A r^{m}\right]_{r e l}$, with a weak change due to Zeeman effect.

Langmuir probe measurements complement these LIF experiments. A Scientific Instruments Langmuir probe was placed in the centre of the diffusion chamber as pictured in Fig. (1). Its working principle and the underlying theory can be found in [36]. To limit the perturbations due to $B_{\text {diff }}$, the tip was aligned to the magnetic field lines [37]. Measured $I(V)$ curves are treated by the Smartsoft software provided with the probe to obtain the electron energy distribution function $(\mathrm{EEDF}) f_{e}(E)$ with:

$$
\int_{0}^{\infty} f_{e}(E) d E=n_{e}
$$

Since Langmuir probe measurements are performed in the centre of the diffusion chamber, i.e. far from the source, EEDF was assumed to be maxwellian. Thus the values for electron density $n_{e}$ and temperature $k T_{e}$ can be deduced from $f_{e}(E)\left(\right.$ in $\left.\mathrm{cm}^{-3} \cdot \mathrm{eV}^{-1}\right)$ :

$$
f_{e}(E)=\frac{2 n_{e} \sqrt{E}}{\sqrt{\pi}\left(k T_{e}\right)^{3 / 2}} \exp \left(\frac{-E}{k T_{e}}\right) .
$$

Fig. (5) presents the experimental EEDFs and their corresponding fit for $P_{R F}=900 \mathrm{~W}$ and $B_{\text {diff }}=10,30$, and $90 \mathrm{G}$. They were determined for energies ranging from 2 to $16 \mathrm{eV}$, the difference between floating and plasma potentials being roughly $15 \mathrm{~V}$. Indeed, at higher energies, measurements become strongly noised and cannot be used any more [38]. By choosing $n_{e}=3.7 \times 10^{10}, 1.6 \times 10^{11}$ and $6.2 \times 10^{11} \mathrm{~cm}^{-3}$, and $k T_{e}=3.9,3.3$ and $2.4 \mathrm{eV}$ respectively, one can see that the maxwellian fit curves are in relatively good accordance with the measurements.

If one considers the $i \rightarrow j$ electron-impact excitation transition for one electron, its average reaction rate coefficient $k_{i j}$ is determined by the convolution of energy dependant electron-impact excitation cross section $\sigma_{i j}(E)$ and electron energy distribution function $f_{e}(E)$ :

$$
\left\langle\sigma_{i j} v_{e}\right\rangle=k_{i j}=\sqrt{\frac{2}{m_{e}}} \cdot \frac{\int_{0}^{\infty} \sigma_{i j}(E) f_{e}(E) \sqrt{E} d E}{\int_{0}^{\infty} f_{e}(E) d E} .
$$


(a)

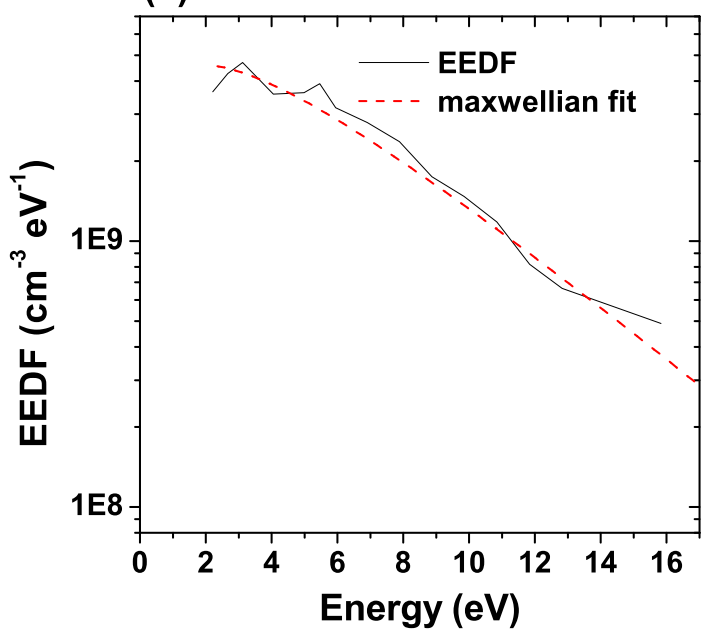

(b)

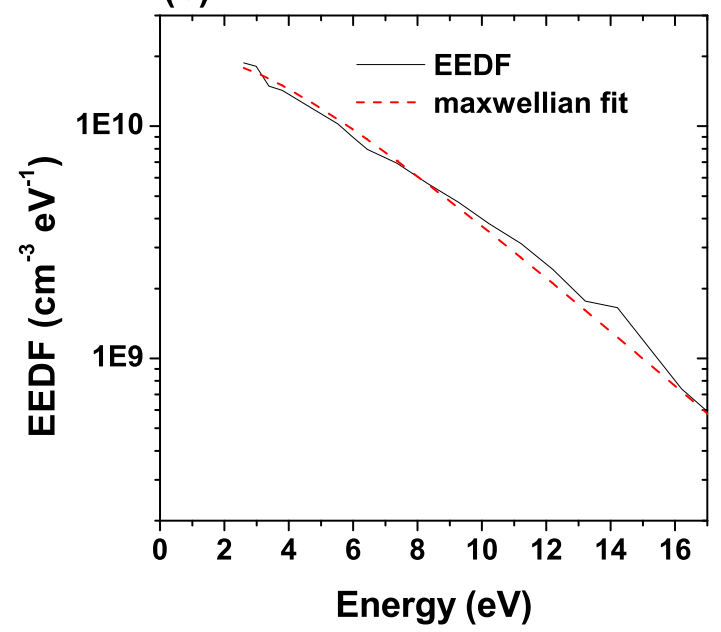

(c)

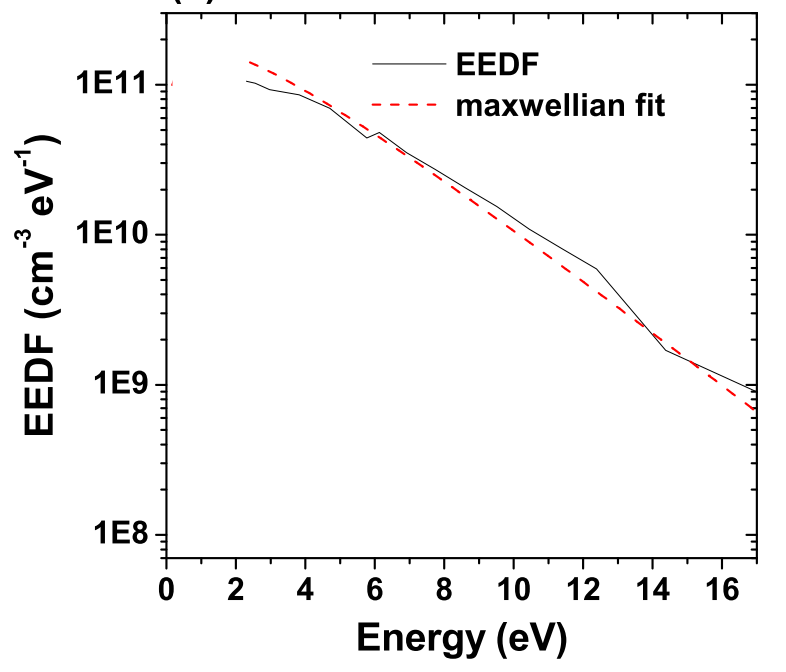

Fig. (5). Measured EEDFs and corresponding Maxwellian fits for $P_{R F}=900 \mathrm{~W}$ and $B_{\text {diff }}=$ (a) $10 \mathrm{G}$, (b) $30 \mathrm{G}$ and (c) $90 \mathrm{G}$.

\section{RESULTS AND DISCUSSION}

\subsection{Selection of $B_{\text {source }}$ and Pressure}

Metastable density depletion can only be observed in inductive mode with a confinement magnetic field $\left(B_{\text {diff }}\right)$ [15]. Fig. (6) presents $n_{e}$, measured in the centre of the diffusion chamber, as a function of pressure with $B_{\text {source }}$ fixed at 0 and $75 \mathrm{G}$, and RF power at $700 \mathrm{~W}$. These experimental conditions permit the observation of all four RF coupling modes available with this plasma source. The behaviour of both curves, which globally decrease with rising pressure, can be explained by two complementary processes. Firstly, increasing pressure reduces the $E / p$ ratio ( $E$ being the electric field and $p$ the pressure) and the electron temperature $T_{e}$, resulting in a diminution of ionization in the source chamber and transport towards diffusion chamber. However, its influence on $n_{e}$ should be progressive and thus does not explain the strong drops observed at 20--50 mTorr and 10--20 mTorr for $B_{\text {source }}=0$ and $75 \mathrm{G}$, respectively. Those drops are characteristic of a mode transition:

- For $B_{\text {source }}=0 \mathrm{G}$, the transition is from inductive to capacitive mode, which results in an important decrease of the electron creation term;

- For $B_{\text {source }}=75 \mathrm{G}$, the drop is steeper, which is typical of the transition from helicon+TG to TG mode. Indeed, this latter transition induces the disappearance of the helicon column along the axis of the diffusion chamber, where the probe measurements are carried out. Eventually, a further pressure increase leads to a transition towards capacitive mode.

Finally, these results show that metastable density depletion is observed when the highest electron density is obtained, which corresponds, in our case, to the inductive coupling $\left(B_{\text {source }}=0 \mathrm{G}\right)$. Thus, this condition was chosen for this study, with a working pressure fixed at $4 \mathrm{mTorr}$. 


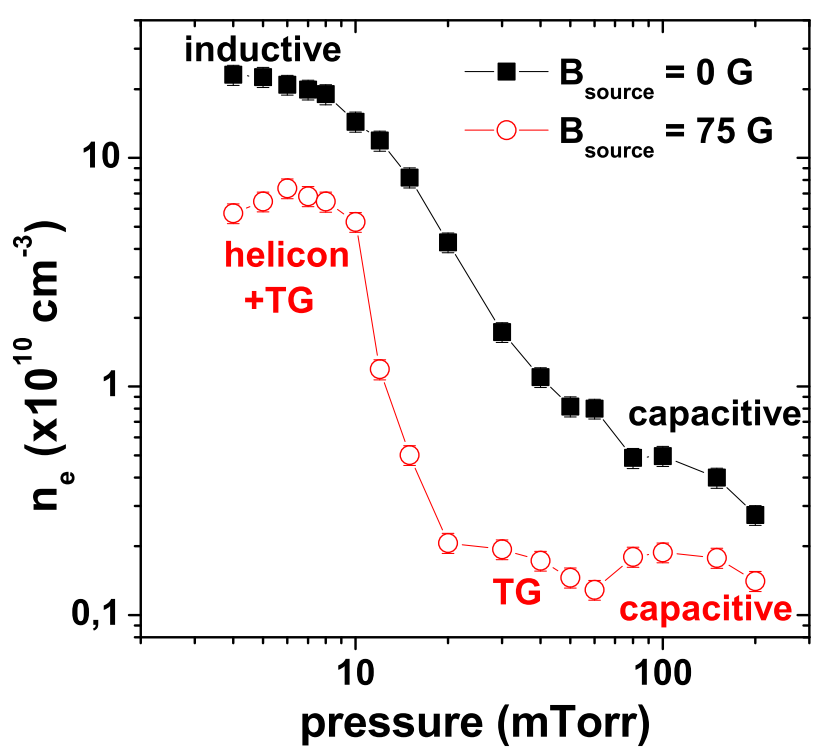

Fig. (6). $n_{e}$ versus pressure with Bsource $=\mathbf{\square} 0 \mathrm{G}$ and $\bigcirc 75 \mathrm{G}, P_{R F}$ $=700 \mathrm{~W}$ and $B_{\text {diff }}=70 \mathrm{G}$.

One can remark that in this case, helicon+TG mode does not exhibit the highest electron density, unlike usual description of helicon sources in the literature. This is due to the fact that, in our conditions, a significant part of the injected power is still transmitted to the plasma trough TG waves at the boundary of the source chamber.

\subsection{Effect of $B_{\text {diff }}$ on $n_{e}$ and $k T_{e}$}

In order to understand the variations of $\mathrm{Ar}$ and $\mathrm{Ar}^{+}$ metastable populations, it is first necessary to determine the electron characteristics in the diffusion chamber centre. Thus, as shown in Fig. (7) for $700 \mathrm{~W}$ of RF power, EEDFs were measured for $B_{\text {diff }}$ varying from 0 to $100 \mathrm{G}$ by step of 10 G. Then, the electron density and temperature were

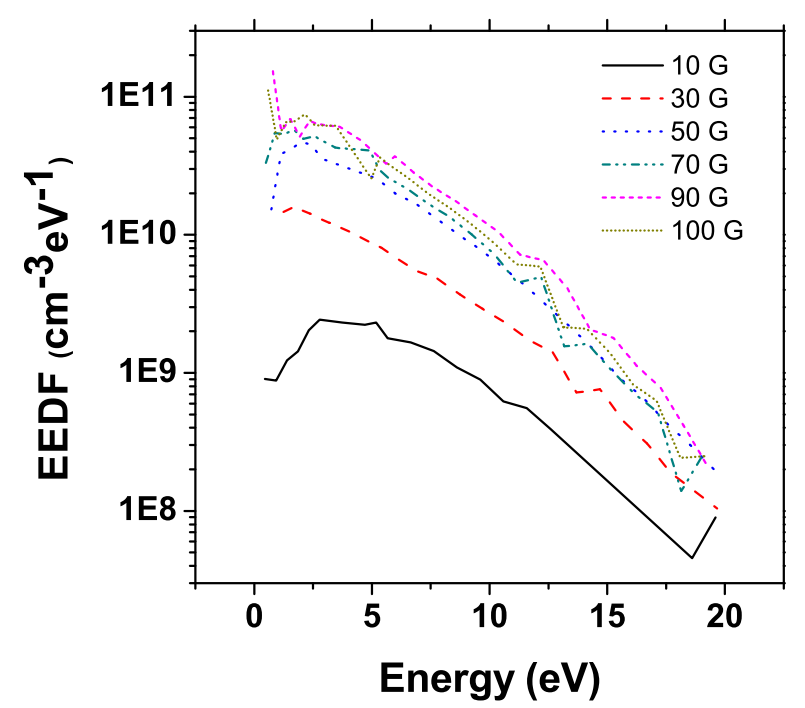

Fig. (7). Evolution of EEDF as a function of $B_{d i f f}$ from 10 to $100 \mathrm{G}$ for $P_{R F}=700 \mathrm{~W}, B_{\text {source }}=0 \mathrm{G}$ and $\mathrm{P}=4$ mTorr. determined by fitting the obtained EEDFs with maxwellian distributions (Fig. $8^{1}$ ). As expected, $n_{e}$ increases with $B_{\text {diff }}$. Indeed, the latter traps the electrons in the vicinity of the diffusion chamber centre, where the magnetic field is the strongest, and thus, reduces electron losses towards the reactor walls.

On the other hand, $k T_{e}$ behaviour is more complicated. It increases with respect to $B_{\text {diff }}$, reaching a maximum at $30 \mathrm{G}$ for $500 \mathrm{~W}, 20 \mathrm{G}$ for $700 \mathrm{~W}$ and $10 \mathrm{G}$ for $900 \mathrm{~W}$. Raising the magnetic field beyond those values, $k T_{e}$ decreases drastically before reaching a plateau where its value is minimal. In inductive mode, plasma is mainly created at the lower loop of the antenna [15], i.e. at the bottom of the source chamber. At this location, $B_{\text {diff }}$ is two times lower than in the centre of the diffusion chamber. It can affect the electron motion by limiting their radial flow, reducing electron losses in the source chamber, and enhancing the axial flow towards the diffusion chamber. Moreover, electron cyclotron resonance, occurring for a magnetic field of $\simeq 5 \mathrm{G}$ for $13.56 \mathrm{MHz}$, could also improve electron heating in the source chamber.

Thus, for low values of $B_{\text {diff }}$, it seems that these electrons coming from the source chamber, more energetic than for $B_{\text {diff }}=0 \mathrm{G}$, could explain the slight increase of $k T_{e}$. The reduction of electron losses still occurs with further raising of $B_{\text {diff }}$ but is covered up by another phenomenon lowering electron temperature. Indeed, the electron gyromotion around the magnetic field lines increases with $B_{\text {diff }}$, inducing fewer electron losses. Their residence time being longer, electrons can suffer more inelastic electron/atom collisions, cooling them in the diffusion chamber. In the end, the maximum value of $k T_{e}$ seems to be the best compromise between the axial transport and inelastic collisions cooling processes.

\subsection{Study of Argon Ion}

First experiments investigated the $3 d^{4} F_{7 / 2} \quad \mathrm{Ar}^{+}$ metastable level. $\left[A r^{+, m}\right]_{r e l}^{668}$ and $k T_{e}$ were measured as a function of $B_{\text {diff }}$ for different RF powers, as depicted in Fig. (9): (a) $500 \mathrm{~W}$, (b) $700 \mathrm{~W}$, (c) $900 \mathrm{~W}$. At low $B_{\text {diff }}$, $\left[A r^{+, m}\right]_{r e l}^{668}$, as $k T_{e}$, increases slightly. One can then observe a drastic augmentation of $\mathrm{Ar}^{+}$metastable population starting when $k T_{e}$ reaches its maximum value, underlined by a full black line on the graphs: at $30 \mathrm{G}$ for $500 \mathrm{~W}, 20 \mathrm{G}$ for $700 \mathrm{~W}$ and $10 \mathrm{G}$ for $900 \mathrm{~W}$. This behaviour is similar to the one of $n_{e}$ in Fig. (8) and occurs until $k T_{e}$ reaches a specific range of values, between around 2.5 and $3.0 \mathrm{eV}$, depending on the conditions. Then, $\left[A r^{+, m}\right]_{r e l}^{668}$ decreases beyond a threshold $B_{\text {diff }}$ value (related to the $k T_{e}$ range of

${ }^{1}$ For clarity, Figs. (9-11) do not present all the error bars (estimated around $\pm 10 \%$ ). 

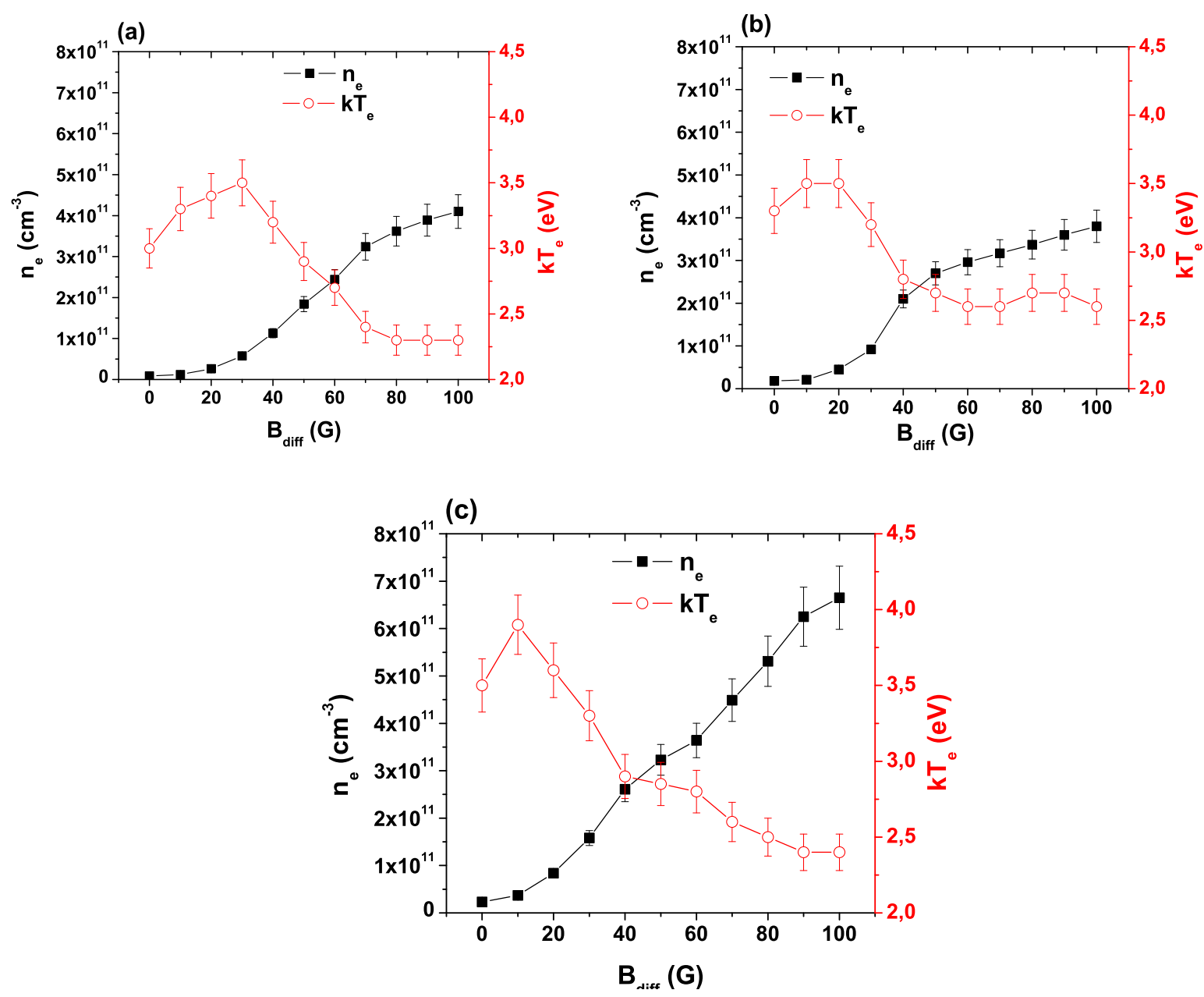

Fig. (8). $n_{e}$ and $\bigcirc k T_{e}$ obtained by fitting the EEDFs with a maxwellian distribution as a function of $B_{d i f f} ; P_{R F}=($ a) $500 \mathrm{~W}$, (b) $700 \mathrm{~W}$ and (c) $900 \mathrm{~W} ; B_{\text {source }}=0 \mathrm{G}$ and $\mathrm{P}=4$ mTorr.

values aforementioned), underlined by a dashed grey line on the graphs: at $60 \mathrm{G}$ for $500 \mathrm{~W}, 40 \mathrm{G}$ for $700 \mathrm{~W}$ and 35 $\mathrm{G}$ for $900 \mathrm{~W}$. This behaviour is contrary to that of $n_{e}$, as seen in Fig. (8). This discrepancy shows that $\left[A r^{+, m}\right]$ is certainly not representative of argon ion density and that experimentally increasing $B_{\text {diff }}$ above a particular value results in a diminution of $\mathrm{Ar}^{+}$metastable population but not of the total $\mathrm{Ar}^{+}$density. Moreover, one can notice that this destruction of $\mathrm{Ar}^{+}$metastable state is observed when $k T_{e}$ decrease becomes slower or not significant.

Previous works [15] presented $k T_{e}$ calculated by the Langmuir probe software (fit of measured $I(V)$ curves) which continuously decreases with $B_{\text {diff }}$. The new determination of $k T_{e}$ by fitting experimental EEDFs gives good correlations between $k T_{e}$ and $\left[A r^{+, m}\right]_{r e l}^{668}$ behaviours.

Moreover, same experiments were made with the OPOLIF diagnostic tuned to measure $3 d^{2} G_{9 / 2}$ relative density $\left[A r^{+, m}\right]_{\text {rel }}^{611}$. The results are shown in Fig. (10). The $\left[A r^{+, m}\right]_{\text {rel }}^{611}$ curves are similar to $\left[A r^{+, m}\right]_{\text {rel }}^{668}$ graphs in Fig. (9), the $B_{\text {diff }}$ thresholds being 70,50 and $40 \mathrm{G}$ for 500,700 and 900 $\mathrm{W}$ respectively. Though, a divergence is perceived for 500 $\mathrm{W}$ beyond the $70 \mathrm{G} B_{\text {diff }}$ threshold where $\left[A r^{+, m}\right]_{r e l}^{611}$ is quasi constant instead of dropping. Once again, $\left[A r^{+, m}\right]_{r e l}^{611}$ stabilization or depletion occurs when $k T_{e}$ decrease is slower or not significant. The fact that this phenomenon also occurs for the $3 d^{2} G_{9 / 2}$ level supports the belief that this density decrease of metastable state is of course not limited to the $3 d^{4} F_{7 / 2} \mathrm{Ar}^{+}$level.

However, the $B_{\text {diff }}$ values corresponding to the $\left[A r^{+, m}\right]_{\text {rel }}$ drop differ depending on the probed levels. Several factors have to be taken into account to explain this discrepancy. By assuming that this depopulation is mainly due to electronimpact excitation towards upper level, the number of available upper levels plays an important role on destruction efficiency. Nonetheless, the most important parameters are the cross sections of these electron-impact interactions. Unfortunately, very few data concerning electron-impact interactions with $\mathrm{Ar}^{+}$metastable levels can be found in the 

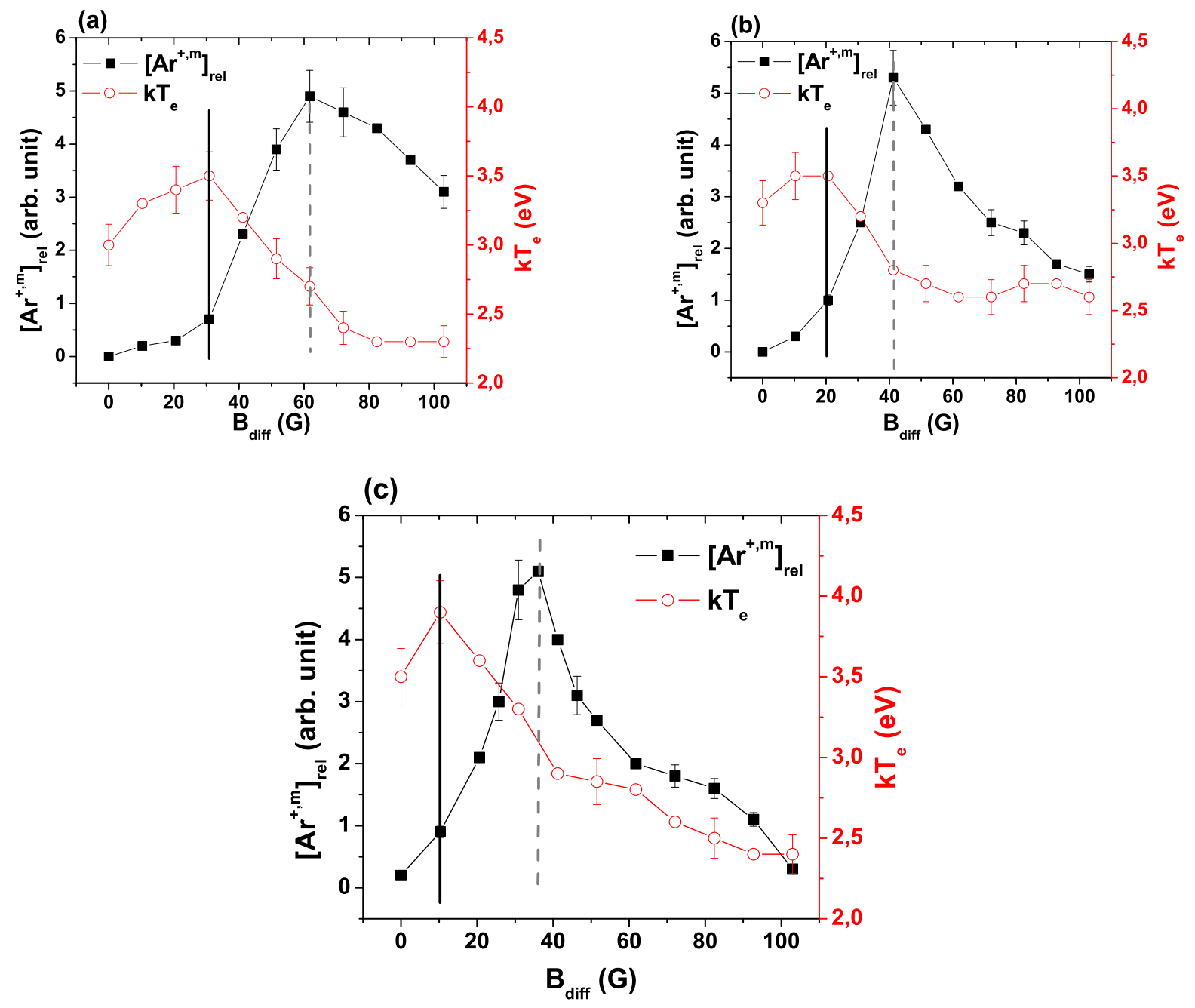

Fig. (9). OPO-LIF at $668.429 \mathrm{~nm}\left(\boldsymbol{\square}\left[A r^{+, m}\right]_{r e l}^{668}\right)$ and $\bigcirc k T e$ measurements versus $B_{\text {diff }} ; P_{R F}=$ (a) $500 \mathrm{~W}$, (b) $700 \mathrm{~W}$ and (c) $900 \mathrm{~W} ; B_{\text {source }}$ $=0 \mathrm{G}$ and $\mathrm{P}=4 \mathrm{mTorr}$.

literature. Consequently, since neutral argon was thoroughly investigated to simulate argon plasmas, the $\left(2 P_{3 / 2}^{0}\right) 4 s^{2}$ neutral argon metastable level was probed in the same experimental conditions to confirm this assumption of destruction by electron collisions and to explain the correlations observed between $\left[A r^{+, m}\right]$ and $k T_{e}$ variations.

\subsection{Study of Neutral Argon}

\subsubsection{Experiments}

Fig. (11) presents $\left[A r^{m}\right]_{r e l}$ and $k T_{e}$ variations versus $B_{\text {diff }}$ for the three RF powers: (a) $500 \mathrm{~W}$, (b) $700 \mathrm{~W}$ and (c) $900 \mathrm{~W}$. The graphs show that $\left[A r^{m}\right]_{\text {rel }}$ exhibits the same behaviour as $k T_{e}$, it increases until a $B_{\text {diff }}$ threshold and drops beyond it. The maximum $\left[A r^{m}\right]_{r e l}$ value is reached at $30 \mathrm{G}$ for $500 \mathrm{~W}, 20 \mathrm{G}$ for $700 \mathrm{~W}$ and $15 \mathrm{G}$ for $900 \mathrm{~W}$. This correlation shows that $k T_{e}$ is the plasma parameter directly linked to neutral metastable state population under our conditions.
Moreover, the conditions where $n_{e},\left[A r^{+, m}\right]_{\text {rel }}^{668}$ and $\left[A r^{+, m}\right]_{r e l}^{611}$ present a drastic increase seem to correspond to the $k T_{e}$ maxima. That is to say when axial transport of energetic electrons, which implies a higher $k T_{e}$ thus an expected higher ionization rate, becomes less important than inelastic collisions, which induces electron cooling and neutral argon metastable state depopulation. One could have expected the ion argon metastable levels densities to present a similar, or even accentuated, behaviour than the neutral argon metastable one, but, the opposite is observed. This phenomenon could be explained by the fact that a significant part of the destroyed $\mathrm{Ar}^{\mathrm{m}}$ must produce ions. This multistep ionization process provides an additional source for $\mathrm{Ar}^{+}$which overcomes, on the one hand, the lower efficiency of direct, i.e. one step, electron impact ionization due to the drop of $k T_{e}$ and, on the other hand, the destruction of $\left[A r^{+, m}\right]$ by electron excitation towards upper levels. When $\left[A r^{m}\right]_{r e l}$ is low enough (underlined by dashed grey lines), multi-step ionization effect becomes less important and ion 

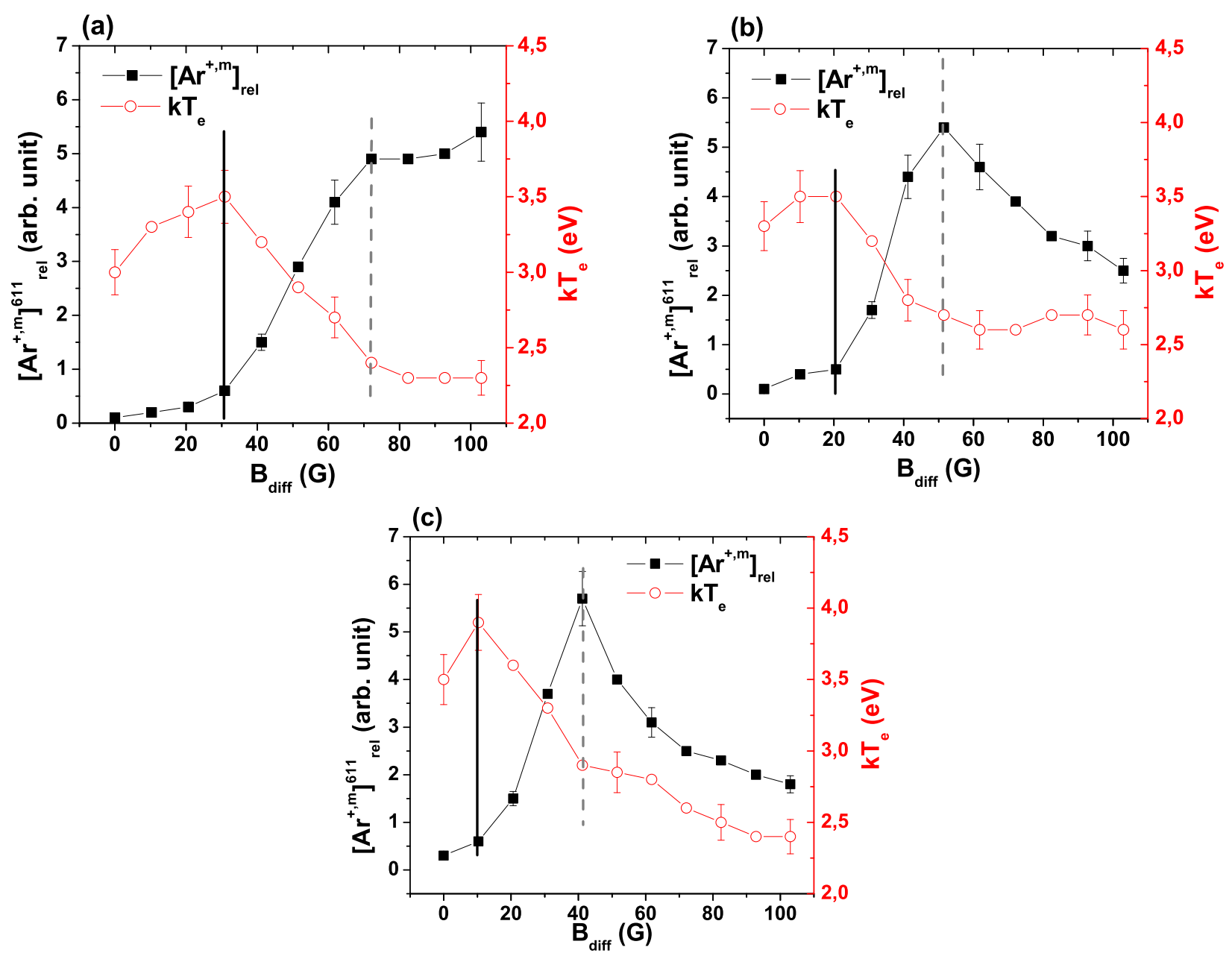

Fig. (10). OPO-LIF at $611.492 \mathrm{~nm}\left(\boldsymbol{\square}\left[A r^{+, m}\right]_{r e l}^{611}\right)$ and $\bigcirc k T_{e}$ measurements versus $B_{d i f f} ; P_{R F}=$ (a) $500 \mathrm{~W}$, (b) $700 \mathrm{~W}$ and (c) $900 \mathrm{~W}$; $B_{\text {source }}=0 \mathrm{G}$ and $\mathrm{P}=4$ mTorr.

metastable population depletion is finally dominant. Thus, the ion metastable levels are destroyed in conditions that present a low neutral metastable density.

\subsubsection{Estimation}

Calculations on neutral argon were developed to support the assumption of metastable levels destruction by electronimpact excitation and to estimate the expected evolution of $\left[A r^{m}\right]_{r e l}$. This estimation considers the $\left(2 P_{3 / 2}^{0}\right) 4 s^{2}$ metastable state. We assume that the argon metastable $1 s 5$ is mainly populated by electron-impact excitation from the ground state (source term $S$ ). The loss mechanisms $L$ are also electron-impact interactions but this time from the metastable to higher energetic levels. The transitions taken into account are presented in Table 1 . The total loss term $L$ includes transitions to several $2 p$ levels, which have the largest cross sections, and ionization, whose relevance is confirmed by experiments presented in this paper. Moreover, when compared with other cross sections taken into consideration, that of the ionization is not to neglect.

Since the plasma is in steady state, $\left[A r^{m}\right]$ follows:
$\frac{d\left[A r^{m}\right]}{d t}=S-L=0$

with:

$$
\begin{aligned}
& S=\left[A r^{g}\right] \times n_{e} \times k_{g m}\left(T_{e}\right), \\
& L=\sum_{i>m}\left(\left[A r^{m}\right] \times n_{e} \times k_{m i}\left(T_{e}\right)\right),
\end{aligned}
$$

where:

- $k_{g m}$ is the reaction rate coefficient to produce the metastable level $m$ from the ground state $g$, and $k_{m i}$ is the one for destruction of $m$ towards upper level $i$ or ionization.

- $\left[A r^{g}\right]$ is the ground state density: about $7.2 \times 10^{13} \mathrm{~cm}^{-3}$ for $P=4 \mathrm{mTorr}$ at around $500 \mathrm{~K}$. The gas temperature was determined by laser absorption spectroscopy on the argon metastable at $801.479 \mathrm{~nm}$. The value grows from 400 to $530 \mathrm{~K}$ between $B_{\text {diff }}=0$ and $200 \mathrm{G}$, respectively. 

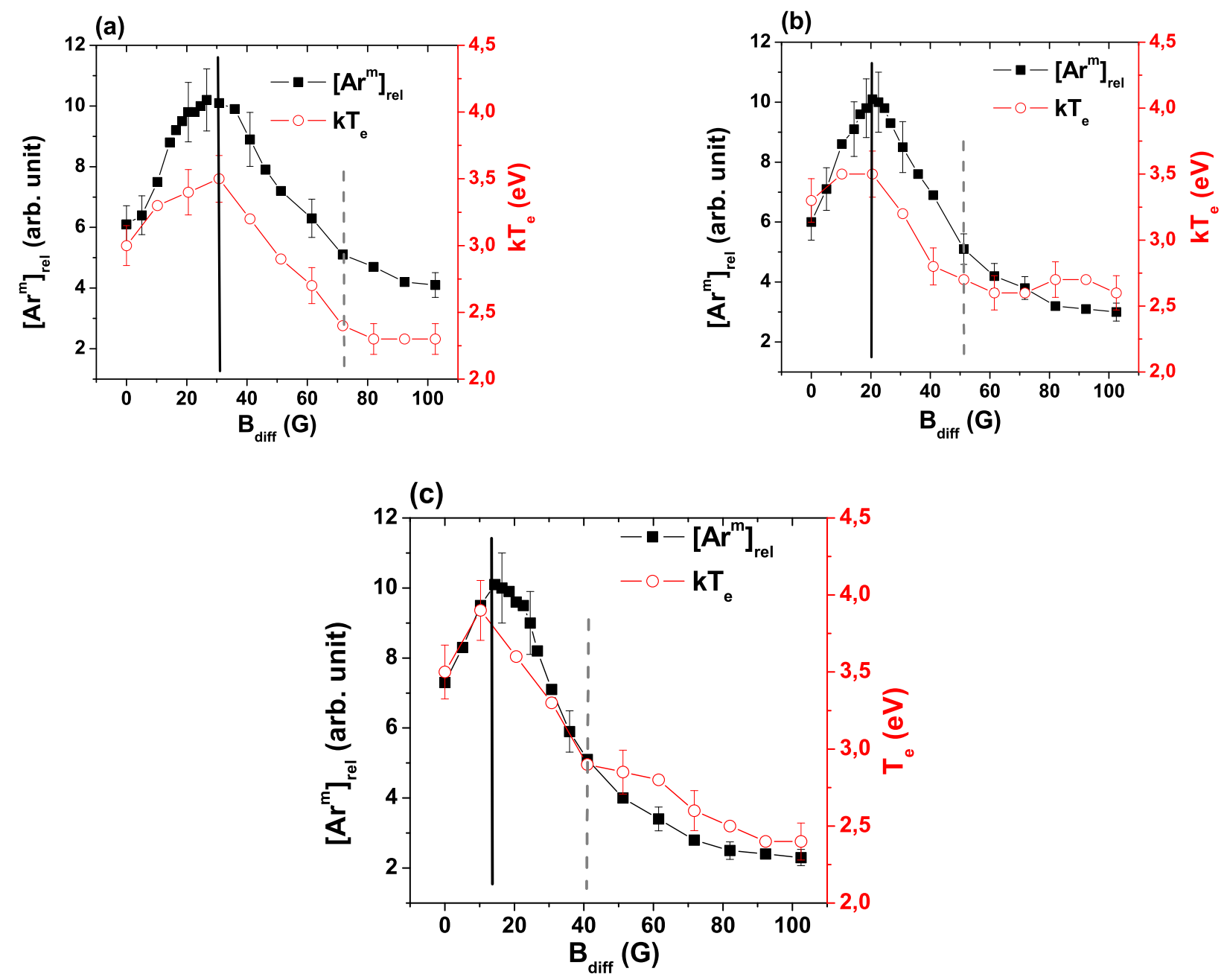

Fig. (11). OPO-LIF at $696.543 \mathrm{~nm}(\boldsymbol{\square}[\mathrm{Arm}] \mathrm{rel})$ and $\bigcirc k T_{e}$ measurements versus $B_{\text {diff }} ; P_{R F}=(\mathrm{a}) 500 \mathrm{~W}$, (b) $700 \mathrm{~W}$ and (c) $900 \mathrm{~W} ; B_{\text {source }}=$ $0 \mathrm{G}$ and $\mathrm{P}=4$ mTorr.

The cross sections corresponding to the loss terms [20, 39], start at $1.528 \mathrm{eV}$ and the energy of the source term cross section maximum is $16 \mathrm{eV}$ [22]. This raises an important issue since EEDF measurements are indeed limited to $20 \mathrm{eV}$ which is inappropriate for the source term. Thus, $n_{e}$ and $k T_{e}$ included in the calculations were determined by their maxwellian fit up to $50 \mathrm{eV}$.

As mentioned above, the terms are calculated as follows:

$$
\begin{aligned}
S & =\left[A r^{g}\right] \times n_{e} \times k_{S} \\
& =\left[A r^{g}\right] n_{e} \sqrt{\frac{2}{m_{e}}} \cdot \frac{\int_{0}^{50} \sigma_{g m}(E) f_{e}(E) \sqrt{E} d E}{\int_{0}^{\infty} f_{e}(E) d E} \\
L & =\left[A r^{m}\right] \times n_{e} \times k_{L} \\
& =\left[A r^{m}\right] n_{e} \sum_{i>m} \sqrt{\frac{2}{m_{e}}} \cdot \frac{\int_{0}^{50} \sigma_{m i}(E) f_{e}(E) \sqrt{E} d E}{\int_{0}^{\infty} f_{e}(E) d E} .
\end{aligned}
$$

Once the source $S$ and loss $L$ terms estimated, due to the plasma steady state, one can deduce the Ar metastable state density:

$$
\left[A r^{m}\right]_{c a l}=\frac{k_{S}}{k_{L}}\left[A r^{g}\right]
$$

Fig. (12) shows the probability of excitation or destruction (per atom) $n_{e} \times k_{S}(\boldsymbol{\square})$ and $n_{e} \times k_{L}(\boldsymbol{\Delta})$, as well as the metastable density $\left[A r^{m}\right]_{\text {cal }}(\bigcirc)$, as a function of $B_{\text {diff }}$ for the three previous RF power values: (a) 500 , (b) 700 and (c) $900 \mathrm{~W}$.

The obtained $\left[A r^{m}\right]_{c a l}$ are in good agreement with the experiments (Fig. 11). $\left[A r^{m}\right]_{c a l},\left[A r^{m}\right]_{r e l}$ and $k T_{e}$ display a similar behaviour with the same $B_{\text {diff }}$ corresponding to a peak value for each power, at $30 \mathrm{G}$ for $500 \mathrm{~W}, 20 \mathrm{G}$ for $700 \mathrm{~W}$ and $10 \mathrm{G}$ for $900 \mathrm{~W}$.

Due to the $B_{\text {diff }}$ confinement, which induces a drastic augmentation of $n_{e}$, one can see that $n_{e} \times k_{S}$ increases 
Table 1. Electron-Impact Excitations Taken into Account in the Model

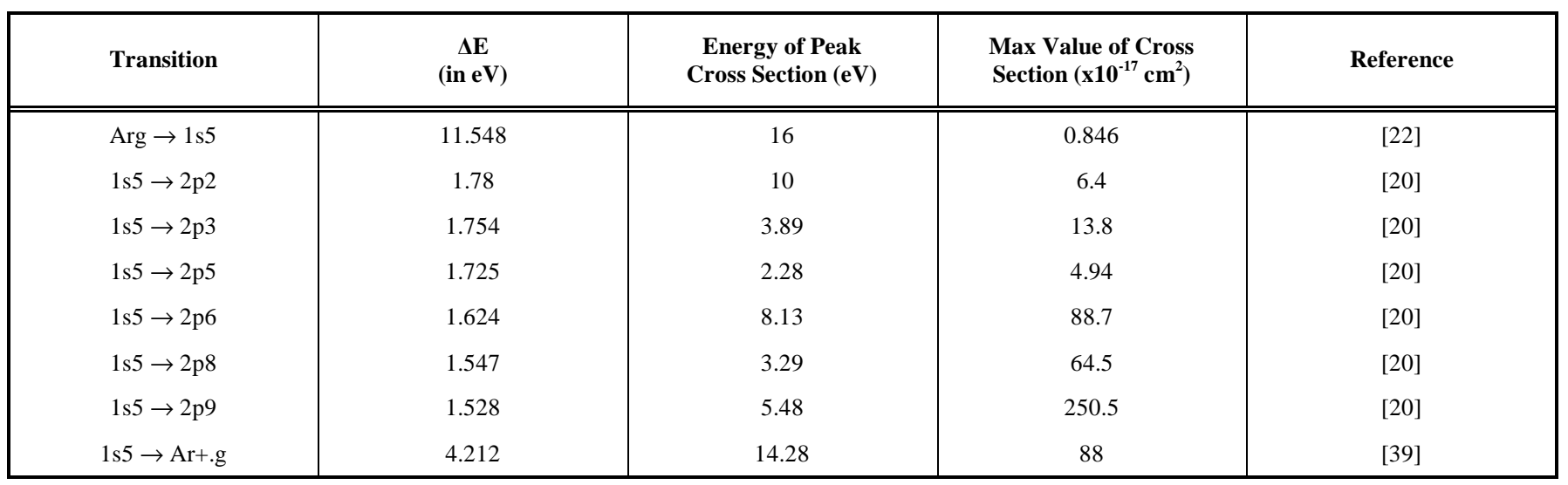
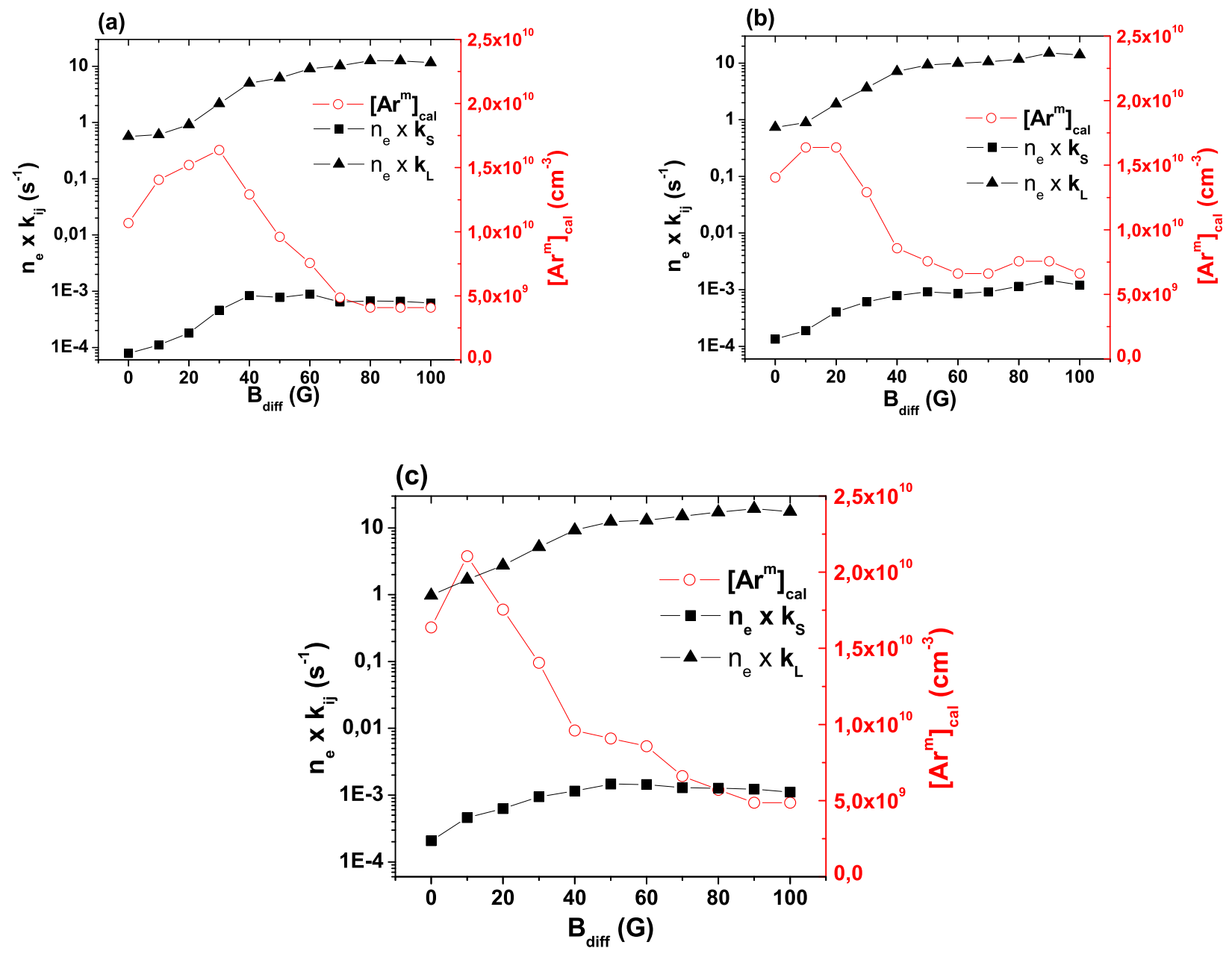

Fig. (12). Calculated number of events $n e \times k_{S}$ and $n_{e} \times k_{L}$, and $\left[A r^{m}\right]_{c a l}$ obtained with the model versus $B_{d i f f}$, for $P_{R F}=($ a) $500 \mathrm{~W}$, (b) $700 \mathrm{~W}$ and (c) $900 \mathrm{~W}$.

around 5 to 10 times with $B_{\text {diff }}$ while $n_{e} \times k_{L}$ gains a factor of around 20. These calculations underline that the influence of $B_{d i f f}$ on electron energy distribution favours destruction processes over creation one by a minimum factor of 2 . Indeed, as showed in Table 1, the creation process needs electrons with at least $11.548 \mathrm{eV}$ of energy while destruction ones only require electrons with energy higher than $1.528 \mathrm{eV}$.

Nevertheless, since no clear relationship between $k T_{e}$ and $n_{e} \times k_{S}$ or $n_{e} \times k_{L}$ can be seen, the reaction rates $k_{S}$ and $k_{L}$ were plotted as a function of $B_{\text {diff }}$ in Fig. (13) $\left(P_{R F}=500\right.$ 
W) to see only the influence of $k T_{e}$. The latter was added to the figure to highlight the correlations.

One can see that while $k_{L}$ remains almost constant, $k_{S}$ is strongly dependant of $B_{\text {diff }}$ and follows $k T_{e}$. The crosssections of these interactions enable the understanding of this behaviour. As previously stated, compared to destruction mechanisms, creation process needs higher-energy electrons to occur. Since $k T_{e}$ variations have a greater impact on the high-energy tail of the EEDF, the creation process is much more sensitive to $k T_{e}$ variations than destruction ones. This phenomenon leads to the observed $k_{S}$ and $k_{L}$ from the Fig. (13). Considering that $\left[A r^{m}\right]_{c a l}$ is function of the reaction rates ratio $k_{S} / k_{L}$, its behaviour is analogous to that of $k T_{e}$.

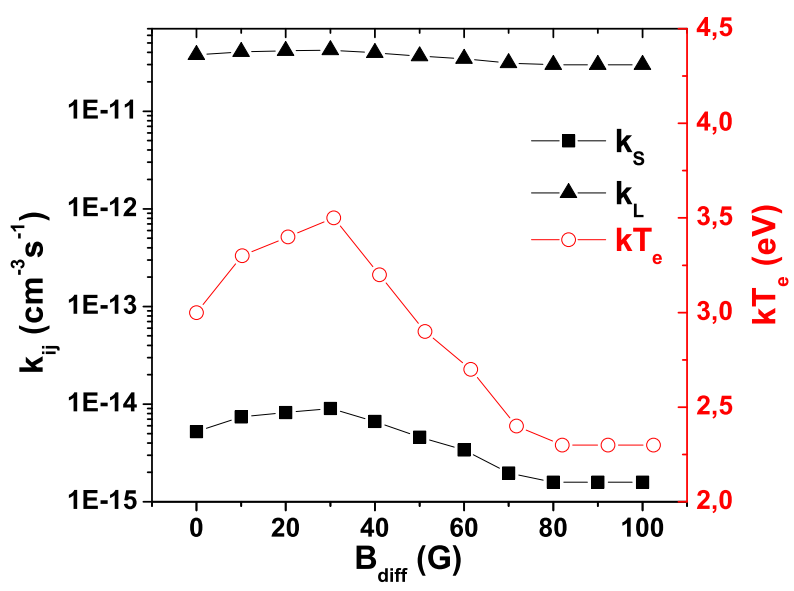

Fig. (13). Calculated reaction rates $k_{S}$ and $k_{L}$, and measured $k T_{e}$ versus $B_{\text {diff }}$ for $P_{R F}=500 \mathrm{~W}$.

By focusing further on $k T_{e}$ behaviour, it is well known that its decay is mainly governed by inelastic collisions. Looking at the energy gaps between possible transitions, it then becomes obvious that the probability of electron-impact interactions to occur is far superior for $A r^{m}$ than for $A r^{g}$. As a result, the main plasma parameters leading the $k T_{e}$ variations are $n_{e}$ and $\left[A r^{m}\right]$. As previously explained, the shape of the $k T_{e}$ curve is a competition between two phenomena: axial transport of high-energy electrons and inelastic collisions. Moreover, $n_{e}$, and thus $\left[A r^{m}\right]$, increase with the confinement magnetic field which permits to reach a significant number of $\left\{A r^{m}-e^{-}\right\}$collisions. Above a certain threshold, those collisions generate the cooling of the electrons bringing to the $A r^{m}$ depopulation. This process could explain the shift in the $B_{\text {diff }}$ value corresponding to the $k T_{e}$ maxima that occurs when changing the RF power. Indeed, a higher RF power induces a rise of the electron and neutral argon metastable densities; consequently, the collisional threshold leading to $k T_{e}$ drop is reached for a lower $B_{\text {diff }}$ value. Eventually, when the neutral argon metastable levels are mainly destroyed, i.e at high $B_{\text {diff }}$, inelastic collisions are not numerous enough to significantly cool the electrons, which leads to the stabilization of $k T_{e}$.

\section{CONCLUSION}

$\mathrm{Ar}$ and $\mathrm{Ar}^{+}$metastable levels destruction was studied in an inductively coupled plasma using a Boswell-type antenna as a function of the confinement magnetic field $B_{d i f f}$, for different fixed RF powers. The densities of these levels were obtained by laser induced fluorescence, and Langmuir probe measurements gave the electron density and temperature deduced from electron energy distribution functions.

Experiments showed that all probed metastable levels are depopulated with increasing confinement magnetic field. It proves that performing LIF experiments on argon in magnetized plasma requires caution, since metastable state density variations do not systematically reflect ground state density behaviour. Indeed, in dense plasmas, i.e. $n_{e}>$ $10^{11} \mathrm{~cm}^{-3}$, the metastable destruction evidenced in [15] is not limited to the $3 d^{4} F_{7 / 2}$ state but can occur with other metastable levels of $\mathrm{Ar}^{+}$and $\mathrm{Ar}$, such as those studied in this work.

$\mathrm{Ar}^{\mathrm{m}}$ density estimations calculated in this paper show that in our conditions, it exhibits a similar behaviour as $k T_{e}$ due to the sensitivity of $\mathrm{Ar}^{\mathrm{m}}$ creation process to $k T_{e}$ variations. On the other hand, $\left[A r^{m}\right]$ seems to be the major parameter influencing $k T_{e}$ through inelastic collisions. It confirms that electron impact interactions are the process leading to this destruction phenomenon. Moreover, it means that $k T_{e}$ behaviour can be determined by means of LIF on neutral metastable. It opens interesting prospects in plasma diagnostics in cases where electrical probe measurements are very difficult to perform.

It was evidenced that a part of the neutral metastable population is ionized, acting as a complementary ionization source. This explains the drastic increase of $\left[A r^{+, m}\right]_{\text {rel }}$ appearing in these conditions and also why $\mathrm{Ar}^{+, \mathrm{m}}$ destruction is observed at high $B_{\text {diff }}$. Indeed, the two-step ionization overcomes the depopulation process, thus, ion metastable states density only decreases when neutral metastable state is considerably depleted. This two-step ionization, superimposed on the $n_{e}$ increase by electron confinement effect when increasing the magnetic field, permits to obtain very dense plasmas in our conditions which can be an asset in plasma-surface interaction studies.

\section{CONFLICT OF INTEREST}

The authors confirm that this article content has no conflict of interest.

\section{ACKNOWLEDGEMENTS}

This work, supported by the European Community under the contract of Association between EURATOM, CEA, and the French Research Federation for fusion studies, was carried out within the framework of the European Fusion 
Development Agreement. The views and opinions expressed herein do not necessarily reflect those of the European Commission. Financial support was also received from the French National Research Agency through contract ANR08-JCJC-0068-01.

\section{REFERENCES}

[1] Cristophorou LG, Olthoff JK. Electron interactions with excited atoms and molecules. Adv At Mol Opt Phys 2001; 44: 155-293.

[2] Kielhbauch MW, Graves DB. Modeling argon inductively coupled plasmas: The electron energy distribution function and metastable kinetics. J Appl Phys 2002; 91: 3539-46.

[3] Stern RA, Johnson III JA. Plasma ion diagnostics using resonant fluorescence. Phys Rev Lett 1975; 34(25) 1548-51.

[4] Burgess DD, Skinner CH. Dye laser induced fluorescence of plasmas and its application to measurement of lower state decay rates. J Phys B At Mol Phys 1974; 7(9): L297-301.

[5] Hebner GA. Spatially resolved, excited state densities and neutral and ion temperatures in inductively coupled argon plasmas. J Appl Phys 1996; 80: 2624-36.

[6] Sadeghi N, Van De Grift M, Vender D, Kroesen GMW, De Hoog FJ. Transport of argon ions in an inductively coupled high-density plasma reactor. Appl Phys Lett 1997; 70(7): 835-7.

[7] Hebner GA, Miller PA. Behavior of excited argon atoms in inductively driven plasmas. J Appl Phys 2000; 87(12): 8304-15.

[8] Scime EE, Keiter PA, Zintl MW, Balkey MM, Kline JL, Koepke ME. Control of ion temperature anisotropy in a helicon plasma. Plasma Sources Sci Technol 1998; 7(2): 186-91.

[9] Luggenhölscher D, Celik Y, Pu YK, Czarnetzki U. Argon ion velocity distributions in a helicon discharge measured by laser induced fluorescence, in Journal of Physics: Conference Series, IOP Publishing 2010; 227(1)-012035.

[10] Sadeghi N, Nakano T, Trevor DJ, Gottscho RA. Ion transport in an electron cyclotron resonance plasma. J Appl Phys 1991; 70(5): 2552-69.

[11] Nakano T, Sadeghi N, Trevor DJ, Gottscho RA, Boswell RW. Metastable chlorine ion transport in a diverging field electron cyclotron resonance plasma. J Appl Phys 1992; 72(8): 3384-93.

[12] de Poucques L, Vitelaru C, Minea TM, Bretagne J, Popa G. On the anisotropy and thermalization of the metal sputtered atoms in a low-pressure magnetron discharge. Europhys Lett 2008; 82(1): 15002.

[13] Vitelaru C, Aniculaesei C, de Poucques L et. al.. Tunable diodelaser induced fluorescence on $\mathrm{Al}$ and $\mathrm{Ti}$ atoms in low pressure magnetron discharges. J Phys D Appl Phys 2010; 43(12): 124013.

[14] Vitelaru C, de Poucques L, Minea TM, Popa G. Time resolved metal line profile by near-ultraviolet tunable diode laser absorption spectroscopy. J Appl Phys 2011; 109(5): 053307.

[15] Bieber T, Bardin S, de Poucques L et. al.. Measurements on argon ion by tunable diode-laser induced fluorescence in a low magnetic field helicon configuration reactor. Plasma Sources Sci Technol 2011; 20(1): 015023 .

[16] Bretagne J, Callede G, Legentil M, Puech V. Relativistic electronbeam-produced plasmas. I. Collision cross sections and loss function in argon. J Phys D Appl Phys 1986; 19(5): 761.

[17] Vlcek J. A collisional-radiative model applicable to argon discharges over a wide range of conditions. I. Formulation and basic data. J Phys D Appl Phys 1989; 22(5): 623.

[18] Bogaerts A, Gijbels R, Vlcek J. Collisional-radiative model for an argon glow discharge. J Appl Phys 1998; 84(1): 121-36.
[19] Bultel A, van Ootegem B, Bourdon A, Vervisch P. Influence of $\mathrm{Ar}^{2+}$ in an argon collisional-radiative model. Phys Rev E 2002; 65(4): 046406.

[20] Boffard JB, Piech GA, Gehrke MF, Anderson LW, Lin CC. Measurement of electron-impact excitation cross sections out of metastable levels of argon and comparison with ground-state excitation. Phys Rev A 1999; 59(4): 2749-63.

[21] Strinić AI, Malović GN, Petrović ZL, Sadeghi N. Electron excitation coefficients and cross sections for excited levels of argon and xenon ions. Plasma Sources Sci Technol 2001; 13(2): 333-42.

[22] Yanguas-Gil A, Cotrino J, Alves LL. An update of argon inelastic cross sections for plasma discharges. J Phys D: Appl Phys 2005; 38(10): 1588-98.

[23] Boffard JB, Chiaro B, Weber T, Lin CC. Electron-impact excitation of argon: Optical emission cross sections in the range of 300-2500 nm. At Data Nucl Data Tables 2007; 93(6): 831-63.

[24] Yanguas-Gil A, Cotrino J, Gonzàlez-Elipe AR. Influence of the excited states on the electron-energy distribution function in lowpressure microwave argon plasmas. Phys Rev E 2005); 72(1): 016401.

[25] Belostotskiy SG, Ouk T, Donnelly VM, Economou DJ, Sadeghi N. Time-and space-resolved measurements of $\operatorname{Ar}\left(1 \mathrm{~s}_{5}\right)$ metastable density in a microplasma using diode laser absorption spectroscopy. J Phys D Appl Phys 2011; 44(14): 145202.

[26] Clarenbach B, Lorenz B, Krämer M, Sadeghi N. Time-dependent gas density and temperature measurements in pulsed helicon discharges in argon. Plasma Sources Sci Technol 2003; 12(3): 34557.

[27] Bieber T, de Poucques L, Vasseur JL, Hugon R, Belmahi M, Bougdira J. Characterization of a neutral atomic hydrogen source developed in the perspective of carbon materials etching study. Surf Coat Technol 2011; 205: S384-7.

[28] Boswell RW. Plasma production using a standing helicon wave. Phys Lett A 1970; 33(7): 457-8.

[29] Arnush D. The role of Trivelpiece-Gould waves in antenna coupling to helicon waves. Phys Plasmas 2000; 7(7): 3042-50.

[30] Lafleur T, Charles C, Boswell RW. Characterization of a helicon plasma source in low diverging magnetic fields. J Phys D Appl Phys 2011; 44(5): 055202.

[31] Severn GD, Edrich DA, McWilliams R. Argon ion laser-induced fluorescence with diode lasers. Rev Sci Instrum 1998; 69(1): 10-5.

[32] Bachet G, Skiff F, Doveil F, Stern RA. Experimental studies of the propagation of electrostatic ion perturbations by time-resolved laser-induced fluorescence. Phys Plasmas 2001; 8: 3535-44.

[33] Gabriel O, Colsters PGJ, Schram DC, Engeln R. Two-dimensional flow characteristic of a hot expanding plasma. Plasma Sources Sci Technol 2008; 17(1): 015011.

[34] Schef P, Derkatch A, Lundin P et. al.. Lifetimes of metastable levels in Ar II. Eur Phys J D 2004; 29: 195-9.

[35] Katori H, Shimizu F. Lifetime measurement of the $1 \mathrm{~s}_{5}$ metastable state of argon and krypton with a magneto-optical trap. Phys Rev Lett 1993; 70: 3545-48.

[36] Tatanova M, Thieme G, Basner R, Hannemann M, Golubovskii YB, Kersten H. About the EDF formation in a capacitively coupled argon plasma. Plasma Sources Sci Technol 2006; 15(3): 507.

[37] Bernstein IB, Rabinowitz IN. Theory of Electrostatic Probes in a Low-Density Plasma. Phys Fluids 1959; 2: 112-21.

[38] Godyak VA, Piejak RB, Alexandrovich BM. Electron energy distribution function measurements and plasma parameters in inductively coupled argon plasma. Plasma Sources Sci Technol 2002; 11(4): 525

[39] Ali MA, Stone PM. Electron impact ionization of metastable rare gases: He, Ne and Ar. Int J Mass Spectrom 2008; 271(1): 51-7. 